\title{
Avaliação do impacto das comorbidades e do tabagismo na sobrevida de pacientes com Leucemia Mielóide Crônica
}

\section{Fernanda F. Engelbrecht*, Kátia B. B. Pagnano}

\section{Resumo}

A Leucemia mielóide crônica (LMC) é uma neoplasia mieloproliferativa da linhagem mielóide, resultante da translocação cromossômica t(9:22); que codifica a oncoproteína BCR-ABL, com atividade tirosinoquinase. Após a introdução dos inibidores de tirosinoquinase houve aumento da prevalência da LMC, devido ao aumento da taxa de sobrevida em 10 anos. Nos casos em que o paciente obtém uma resposta molecular maior, a sobrevida iguala-se a da população em geral. Desse modo, é necessário avaliar outros fatores que afetam a sobrevida da população em geral e a sua relação com o prognóstico de pacientes com LMC. Esse estudo visa analisar o impacto das comorbidades e do tabagismo no prognóstico e na sobrevida dos pacientes com LMC tratados no Hemocentro da UNICAMP. Após coleta e analise de dados de 269 pacientes constatamos que a presença de comorbidades clínicas acarretou diminuição na sobrevida dos pacientes sendo relevante o diagnóstico e controle das mesmas para aumento da qualidade e sobrevida de pacientes com LMC.

Palavras-chave: Leucemia mielóide crônica, Tabagismo, neoplasias secundárias

\section{Introdução}

A Leucemia mielóide crônica (LMC) é uma neoplasia mieloproliferativa da linhagem mielóide, resultante da translocação cromossômica t(9:22); que codifica a oncoproteína BCR-ABL, com atividade tirosinoquinase. Após a introdução dos inibidores de tirosinoquinase (ITK) houve aumento da prevalência da LMC, devido ao aumento da taxa de sobrevida em 10 anos, aproximandose da população normal. Desse modo, torna-se necessário avaliar outros fatores que afetam a sobrevida da população em geral e a sua relação com o prognóstico dos pacientes com LMC. Esse estudo visa analisar o impacto das comorbidades, avaliadas pelo score de Charlson e do tabagismo no prognóstico e na sobrevida dos pacientes com LMC tratados no Hemocentro da UNICAMP.

\section{Resultados e Discussão}

Foram analisados 269 pacientes com LMC diagnosticados entre Janeiro de 2001 a Dezembro de 2018 e tratados com ITK. As caracteristicas clinicas e evolutivas estão resumidas na tabela 1. A sobrevida global foi inferior nos pacientes com mais comorbidades clinicas (figura 1). Houve mais casos de neoplasias secundárias entre os tabagistas ( $50 \%$ nos casos de tumores de próstata, 33\% tumores de pulmão e $17 \%$ no melanoma de coroide).

\section{Conclusões}

A presença de comorbidades clínicas acarretou diminuição na sobrevida dos pacientes, sendo relevante o diagnóstico e controle das mesmas para aumento da qualidade de vida e sobrevida dos pacientes com LMC. O tabagismo parece não afetar o comportamento da LMC, porém pode contribuir para aparecimento de neoplasias secundárias e agravamento das comorbidades, devendo ser evitado.

Bower H, Björkholm M, Dickman PW, et al: Life expectancy of patients with chror myeloid leukemia approaches the life expectancy of the general population. $\mathrm{J}$ Clin Oncol 34:2851-2857, 2016.

Charlson ME, Pompei P, Ales KL, MacKenzie CR. A new method of classifying Figura 1. Sobrevida global de acordo com score de

prognostic comorbidity in longitudinal studies: development and validation. J Chrocharlson

Dis. 1987;40:373-83.

Lauseker, M., Hasford, J., Saussele, S., et al. (2017). Smokers with chronic myeloid

leukemia are at a higher risk of disease progression and premature death. Cancer.

https://doi.org/10.1002/cncr.30636
Tabela1.Características clinicas de pacientes com LMC

\begin{tabular}{|l|l|}
\hline & $\mathrm{n}(\%)$ \\
\hline Idade, mediana, range & $49,(14-86)$ \\
\hline Sexo, masculino & $152(56,5)$ \\
\hline Fase doença ao dx & \\
crônica & $259(96,3)$ \\
acelerada & $10(3,7)$ \\
\hline Escore de Sokal & \\
baixo & $95(35,3)$ \\
intermediario & $81(30,1)$ \\
alto & $78(29)$ \\
NA & $15(5,6)$ \\
\hline Escore de Charslon & \\
2 & $128(47,6)$ \\
3 & $52(19,3)$ \\
4 & $44(16,4)$ \\
$>=5$ & $45(16,7)$ \\
\hline ITK & 269 \\
imatinibe & $268(99,6)$ \\
dasatinibe & $01(0,4)$ \\
\hline Tabagismo (sim) & $91(33,8)$ \\
\hline Comorbidades (sim) & $148(55)$ \\
\hline Progressão doença (sim) & $39(14,5)$ \\
\hline Neoplasia secundária & $21(7,8)$ \\
\hline Óbitos & $57(21,2)$ \\
relacionados a LMC & $25(44)$ \\
\hline
\end{tabular}

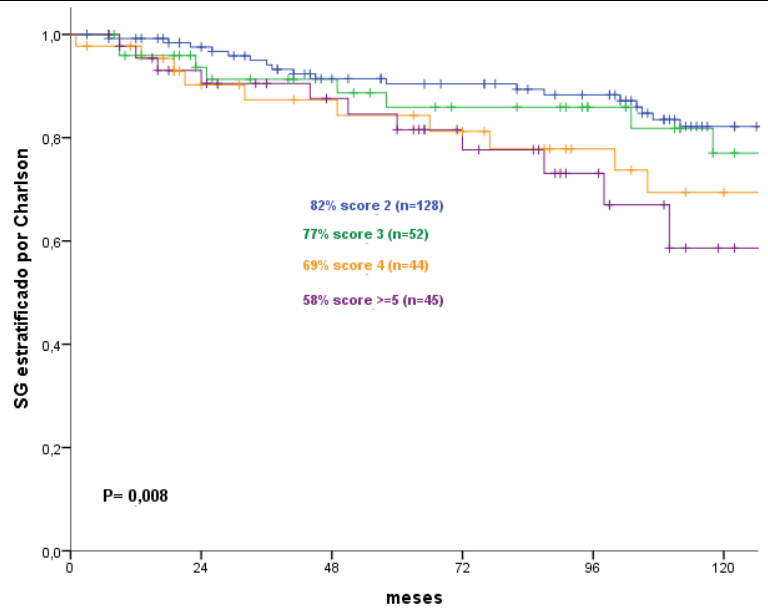

